\title{
10 Jahre zfhr - eine österreichische Erfolgsgeschichte
}

Online publiziert: 22. Februar 2012

(c) Springer-Verlag 2012

Sehr geehrte Damen und Herren,

ich darf Sie im Namen des Verlags Springer WienNewYork sehr herzlich zum Symposium „10 Jahre Zeitschrift für Hochschulrecht/zfhr“ begrüßen!

„Zehn Jahre zfhr - eine österreichische Erfolgsgeschichte" ist ein dankbares Thema, denn es liegt geradezu in der Natur von Verlegerinnen und Verlegern, sehr gerne über Erfolge zu reden. Das tun sie aber nicht aus Eitelkeit, sondern weil Erfolge wirtschaftliche Größen darstellen, weil sie für ein Unternehmen, natürlich auch für unseres, und unsere Autoren äußerst wichtig sind - und weil uns alle Erfolge einfach sehr freuen.

Erfolge fallen einem aber nicht in den Schoß, sondern man muss sie sich erarbeiten, und wenn die richtigen Kräfte, die richtigen Menschen zusammentreffen, kann es funktionieren. Vor zwölf Jahren war das der Fall: Begonnen hat alles, wie so oft in unserer Branche, mit einer Idee und einem Konzept. Herr Prof. Werner Hauser von der FH Joanneum in Graz erarbeitete gemeinsam mit Herrn Prof. Manfred Novak von der Universität Linz und Herrn Dr. Mario Kostal eine inhaltliche Struktur für ein wissenschaftliches Periodikum, das sich mit dem Hochschulrecht auseinandersetzen sollte - und das nicht ohne Grund: Die Zeitschrift „Recht der Schule“ aus dem Manz Verlag war Mitte der 1980er Jahre eingestellt worden, und ab 1993 waren die Hochschulen mit einer Reihe von Gesetzesnovellen und Neukodifizierungen konfrontiert, sodass ein Medium, das sich wissenschaftlich mit der Materie auseinandersetzte, geradezu notwendig geworden war. Mehrere österreichische Verlage zeigten Interesse an diesem Konzept, Springer WienNewYork war aber schneller als die Konkurrenz, und schon im Herbst 2000 wurde verhandelt.

Danach wurde geplant und vor allem gerechnet. Wie hoch sind die Herstellkosten? Wie groß ist die Zielgruppe? Oft kommen wissenschaftliche Publikationen ohne finanzielle Förderungen nicht aus. Herrn Prof. Hauser ist es aber gelungen, das Bundesministerium für Wissenschaft und Forschung und die FH Joanneum von der Wichtigkeit und der Güte dieser Zeitschrift zu überzeugen. Bei diesen beiden Fördergebern sollte es aber nicht bleiben. In den folgenden Jahren kamen noch die Österreichische Fachhochschulkonferenz und die Österreichische Rektorenkonferenz als Förderer hinzu. Der Weiterbestand der zfhr war somit gesichert. Ich möchte mich an dieser Stelle sehr herzlich für dieses Engagement bedanken, nämlich bei Herrn Generalsekretär Mag. Friedrich Faulhammer vom Bundesministerium für Wissenschaft und Forschung, beim Rektor der FH Joanneum, Herrn Prof. Karl Pfeiffer, beim Kaufmännischen Geschäftsführer der FH Joanneum, Herrn Dr. Günter Riegler, beim Generalsekretär der Fachhochschulkonferenz, Herrn Mag. Kurt Koleznik, und beim Präsidenten der Rektorenkonferenz, Herrn Prof. Hans Sünkel. Wir hoffen, dass Sie alle der zfhr auch in Zukunft so gewogen sein werden, denn die zfhr hat ihre Wichtigkeit längst unter Beweis gestellt:

Sie hat sich von Anfang an so wichtiger Themen wie der Hochschulreform, der Universitätsautonomie und akademischen Selbstverwaltung, der Qualität und Kompetenzverteilung von Fachhochschul-Trägervereinen, also Themen aus Recht, Management und Politik angenommen. Heute ist die zfhr eine anerkannte und bei den Lesern hoch geschätzte Zeitschrift, die - insbesondere durch die Möglichkeiten des elektronischen Publizierens - eine immer größere Verbreitung findet. Sie ist seit vielen Jahren in allen wichtigen österreichischen Rechtsdatenbanken abrufbar und darüber hinaus auch via SpringerLink, der Content-Platform von Springer, weltweit zugänglich. 
Natürlich gibt es über eine so lange Zeit immer wieder personelle Veränderungen: Prof. Hauser etwa entschloss sich, seine Funktion als Herausgeber und Schriftleiter zurückzulegen (wobei er der zfhr aber weiterhin - und nicht nur als Autor - eng verbunden blieb), Frau Prof. Bettina Perthold-Stoitzner trat seine Nachfolge an. Dr. Mario Kostal musste seine Funktion als Herausgeber und Schriftleiter aus gesundheitlichen Gründen zurücklegen, ihm folgte Frau Prof. Eva SchulevSteindl nach.

Im Jahr 2009 wurde auch entschieden, die Schriftleitung von der Herausgeberschaft zu trennen und die Schriftleitung zu erweitern, um die Bereiche EU, Hochschulmanagement und Pädagogische Hochschulen in Zukunft verstärkt abzudecken.

Für Kontinuität sorgte in dieser Umbruchphase Herr Prof. Novak, der von Anfang an mit an Bord war und somit einen ganz wesentlichen
Beitrag zur Erfolgsgeschichte der zfhr leistete. Ich darf den Herausgebern, den Schriftleitern und dem wissenschaftlichen Beirat an dieser Stelle für ihr - wohlgemerkt ehrenamtliches Bemühen für die zfhr danken!

Ich hoffe, dass die nächsten zehn Jahre für die zfhr genauso erfolgreich sein werden, wie es die vergangenen zehn waren. Und wenn ich heute einen Wunsch äußern darf, dann den, dass uns unsere Fördergeber auch weiterhin auf unserem Weg begleiten mögen. Ohne Ihre Mittel wäre es uns nicht möglich, die Zeitschrift für Hochschulrecht zu verlegen. Ich bitte Sie daher, dieses wichtige Projekt auch weiterhin zu unterstützen, so dass wir gemeinsam an den Erfolg der vergangenen zehn Jahre anknüpfen und diesen in der Zukunft fortführen können.

Geschäftsführerin Mag. Katharina Oppitz 